\title{
XVII. \\ Zur Genese des Angioma cavernosum.
}

(Aus der chirurgisehen Abtbeilung des James Armitstead'schen Kinder hospitals zu Riga. Dirig. Arzt: Dr. P. Klemm.)

Von

Dr. med. Felix Pilzer, Assistenten.

(Hierzu Taf. VII:)

Das Angioma cavernosum ist eine Geschwulstform, die mit allen übrigen wahren Geschwülsten das Schicksal theilt, in mehrfacher Hinsicht noch keine sichere Erklärung gefunden zu haben. Während wir bei den Granulations-Geschwülsten die Frage: wodurch entstehen sie, wie entstehen sie, in welcher Weise erfolgt ihr Wachsthum, in befriedigender $W$ eise beantworten können, vermögen wir die Definition des cavernösen Angioms nur in morphologischer Hinsicht zu geben, d. h. zu beschreiben, wie es sich dem Auge darstellt, und müssen uns begnügen, mit dem sich daraus ergebenden Begriff, die Frage nach dem Wesen dieser Geschwulst zu beantworten.

Von diesem letzteren Gesichtspunkte aus verstehen wir heute unter dem Angioma cavernosum eine pathologische Neubildung, welche aus zahlreichen blutführenden Hohlräumen und einem diese verbindenden interstitiellen Gewebe besteht.

Die Bezeichnung cavernöse Geschwulst, welche von Meckel, unter Hinweis auf die Aehnlichkeit mit dem Gewebe des physiologischen Corpus cavernosum penis beziehungsweise clitoridis, aufgebracht wurde, ist insofern glücklich gewählt, als sie mit einer ganzen Reihe vorher existirender Namen aufräumte, welche, unter Benutzung aller möglicher, nur sehr oberflächlicher Aehnlichkeiten, einen einheitlichen Begriff nicht hatten aufkommen lassen.

Durch die Förderung, welche das Verständniss dieser Geschwulst durch die Verleihung eines keine Missverständnisse mehr zulassenden Namens erfuhr, ist indessen, wie gesagt, unsere Kenntniss von der Entstehungs-Ursache und -Art nicht wesent- 

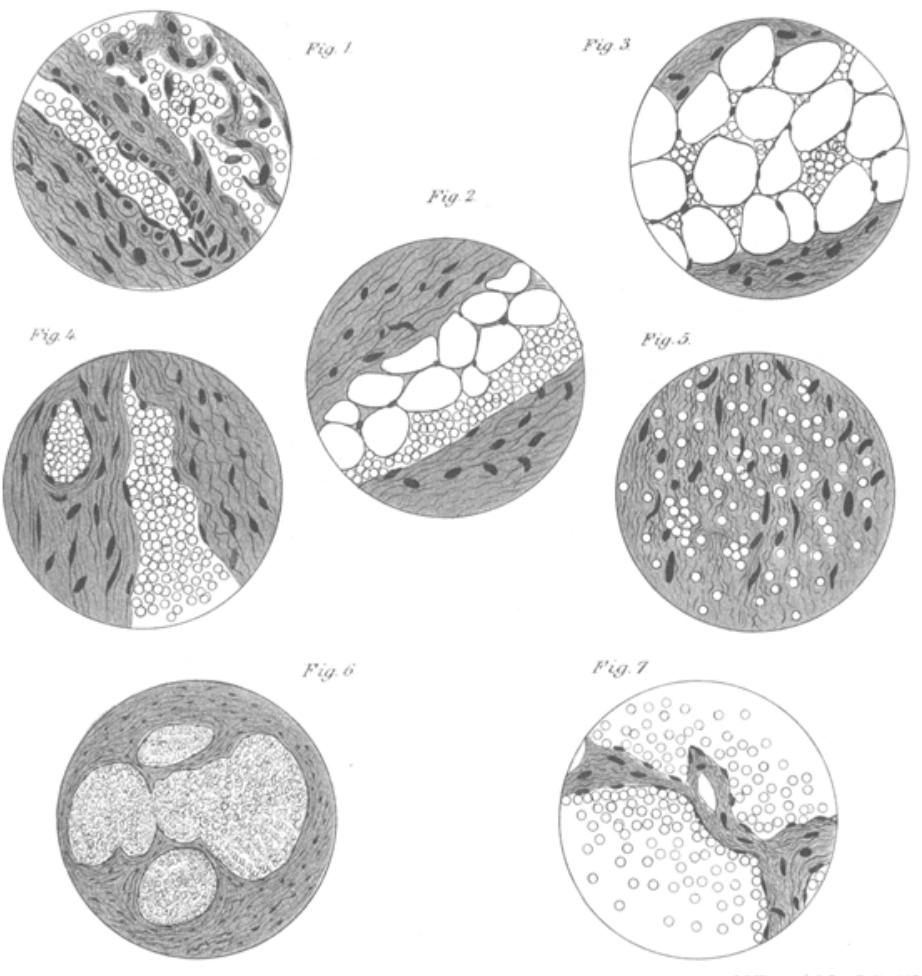


\section{8}

lich beeinflusst worden. Wir stehen hier noch heute vor einem Räthsel, das der Lösung harrt und einstweilen nur zur Aufstellung mehrerer Hypothesen Veranlassung gegeben hat, von denen indessen keine einzige vermocht hat, sich allgemeine Anerkennung zu verschaffen.

Bevor ich die bis jetzt bekannt gewordenen Ansichten über die Entstehung und das Wesen des cavernösen Angioms gegen einander abwäge, möge es mir vergönnt sein, einen in der chirurgischen Abtheilung des hiesigen Kinderhospitals zur Behandlung gekommenen Fall von Angioma cavernosum femoris et cruris zu schildern, welcher in Folge der ausserordentlichen Ausdehnung, welche die Geschwulst erreicht hatte, zu den seltensten Vorkommnissen gehört.

Benze, G., 14 Jahre alt, stamnt aus gesunder Familie, hat in früher Jugend Masern durchgemacht, ist sonst stets gesund gewesen. Von der Geburt an bestanden am linken Knie schwarze Flecken, die ihm keinerlei Beschwerden bereitet hatten, bis zum 12. Jahre. Damals stellten sich im Knie und in der Wade starke Schmerzen ein, die den Patienten veranlassten, in der Ambulanz des "Rothen Kreuzes" ärztliche Hülfe aufzusuchen. Patient wurde bier operirt. Danach sebwanden die Schmerzen und Patient konnte das Bein ohne Beschwerden gebrauchen. Vor $1 \frac{1}{2}$ Monaten bemerkte Patient, dass er sein linkes Bein im Kniegelenk nicht mehr strecken konnte. Er begab sich in Folge dessen in das hiesige Hospital.

Bei seiner am 5. Mai 1900 erfolgten Aufnahme bietet Patient folgenden Befund dar:

Kräftiger Ernährungszustand. An den unteren Extremitäten mehrere alte, bis Handteller-grosse Verbrennungsnarben. Die Haut des Rumpfes, Gesichtes und der Arme ist rein, von gesunder Farbe. Keine Drüsenschwellungen. Brust- und Bauchorgane ergeben durchweg normalen Befund.

Das linke Bein ist im Kniegelenk in einem Hlexionswinkel von ca. $120^{\circ}$ fixirt, lässt sich passiv in einer Ausdehnung von ca. $5^{\circ}$ weiter flectiren, aber nicht strecken. Diese Bewegungsversuche sind schmerzbaft. An der Wade, von der Kniekeble abwärts, verläuft eine ca. $10 \mathrm{~cm}$. lange, schmale Operationsnarbe. In der Gegend des Kniegelenks, an der Aussenseite, trägt die Haut mehrere Naevi vasculosi von Erbsen- bis 10 Kopekenstück-Grösse und bläulich-brauner Farbe. Von dieser Gegend ab nach unten ziehen einige anscheinend dilatirte Venen. Unter der Haut fühlt man mehrere, bis Haselnuss-grasse harte Knoten und Stränge, die, anscheinend in Unterhautzellgewebe sitzend, verschieblich und auf Druck etwas schmerzhaft sind.

Patient hinkt beim Gehen entsprechend der durch die Flexionsstellung bedingten Verkürzung seines Beines und hat beim Gehen geringe Schmerzen. Stuhlgang : normal. Urin: 1022, klar, sauer, kein Albumen. 
Patient erbält zunächst eine Gewichts-Extension un das Bein, sowie einen Sandsack aufs Knie, um eine Streckung des Beines zu bewirken. Diese Streckung ist nach 10 Tagen erreicht.

Es wird nunmehr am 20. Mai zur Operation geschritten (Dr. P. Klemm).

Narkose, Esmarch'scher Schlauch. Längsschnitt an der Hinterseite des linken Oberschenkels von der Kniekehle aus nach oben. Abpräpariren der Haut. Man hat das Unterhautzellgewebe vor sich, welches in grosser Ausdehnung von theils schwammigen, theils derben Knoten durchsetzt ist, deren Schnittflächen aus zahlreichen kleineren und grösseren Oeffnungen schwarzrothes Blut entleeren. Indem diese Knoten bis unter die obersten Hautschichten reichen, bilden sie die bei der äusseren Betrachtung auffallenden bläulichen Flecken. Alle diese Knoten werden exstirpirt, d. h. das ganze Unterhautzellgewebe, welches den Sitz dieser Knoten bildet. Man hat nunmehr die Mrsculatur der Beugeseite des Oberschenkels vor sich. Es erweist sich, dass die Knotenbildung sich auch auf die Musculatur fortgesetzt hat. Der Musculus Biceps fem. hat seine Eigenschaft als Muskel fast vollständig verloren, und besteht zum grössten Theil aus kleinen, bis stecknadelkopfgrossen Lumina, aus denen sich Blut entleert. Zwischen diesen Lumina befindet sich derbes Bindegewebe. Der Muskel bildet hier eine unregelmässige, derbschwammige Geschwulst, die im Querschnitt das normale Maass des Muskels bedentend äbertrifft. Entsprechend dieser Querschnitts-Vergrösserung ist der Muskel verkürzt, und damit augenscheinlich die Ursache zur Flexionsstellung des Kniegelenks. Das ganze so entartete Stück des Muskels, etwa $\frac{3}{3}$ desselben, wird exstirpirt. Unter dem Muskel geht die Geschwulstbildung immer noch weiter und macht erst an der äusseren Seite des Os femur Halt, welches schliesslich, nach Entfernung aller erkrankten Partien, in einer Ausdehnung von ca. $10 \mathrm{~cm}$, vom Condyl. ext. an nach oben gerechnet, frei liegt.

Es werden weitere kleinere Geschwulstmassen entfernt aus der Kniekehle, aus dem Muse. Adductor magnus. Dicht unterhalb des Adductorenschlitzes ist ein Gefässast sichtbar, welcher aus der Vena femoralis in die zunächst gelegenen Geschwulstknoten führt. Der Ast wird unterbunden und durchschnitten.

Unterhalb des Kniegelenks erscheint der Nerv. cruralis gleichfalls in einen derbschwammigen, blauschwarzen Strang verwandelt. Er wird entsprechend seiner Veränderung excidirt.

Um alle Geschwulstmassen zu exstirpiren, ist eine Erweiterung des Schnittes nach unten wie nach oben und am Oberschenkel in querer Richtung nöthig geworden, so dass das gesammte Operationsgebiet schliesslich das obere Drittel der Wade, die äussere Seite des Kniegelenks, sowie die ganze untere, hintere Hãlfte des Oberschenkels umgreift, und rings umrandet wird von der zurückpräparirten und zurückgeschlagenen Haut. Aus dieser werden alle von den Naevi eingenommenen Stellen excidirt.

Nach Lockerung des Schlauches erfolgt eine nicht übermässig starke 
venöse Blutung aus zahlreichen, restirenden, dilatirten Gefässen. Unterbindung derselben. Tamponade der ganzen Wundfäche mit steriler Gaze. Anlegen einiger fixirender Hautnähte über den Tampons. Comprimirender Schienenverband um die ganze untere Extremität. Hochlagerung derselben.

Patient hat während der ganzen, über eine Stunde dauernden Operation kaum mehr als $\frac{1}{8}$ Liter Blut verloren.

Aus dem Krankheitsverlauf ist Folgendes hervorzuheben:

21. Mai. Patient hat die Operation gut überstanden, fühlt sich wohl. Keine Teroperatur-Steigerung.

25. Mai. Die durch die Fixationsnähte äber den Tampons zusammengezogene Haut ist nirgends nekrotiseh geworden. Das ganze Operationsgebiet ist rein, trocken und granulirt gut. Tamponade. Schienenverband.

30. Mai. Von den Hauträndern und vom Grunde der Wundfäche her gute Granulations-Bildung. Das Bein steht in guter, gestreckter Stellung.

5. Juli. Das Wundgebiet verkleinert sich fortgesetzt. Patient vermag im Knie- und Fussgelenk Bewegungen auszuführen.

13. Juli. Narkose. Vom rechten Oberschenkel her werden nach Thiersch Hautlappen auf die Wundfäche des linken Beines verptianzt. Es wird u. A. die ganze Kniekehle wit solehen Lappen versorgt.

17. Juli. Die transplantirten Hautlappen sind der Hauptsache nach angeheilt und zeigen an ibren Rändern gutes Wacbstbum.

30. Juni. Fortschreitende Ueberhäutung. Patient stebt auf und vermag mit Krücken leidlich gut zu gehen.

14. Juli. Patient ist in den letzten 14 Tagen täglich gebadet und verbunden worden, ist bei sehr gutem Befinden. In der Kniekehle ist alles überhäntet. Die Epidermis fehlt noch an einzelnen, nicht sehr ausgedehnten Stellen an der Hinterseite des Unterschenkels, sowie an der Aussenseite des Kniegelenks, hier in einem Umkreise von ca. $5 \mathrm{~cm}$ Durchmesser.

27. Juli. Das linke Bein steht in geringer Varus-Stellung. An der Hinterseite des Oberschenkels ein schmaler Granulations-Streifen. An der Aussenseite des Kniegelenks Epidermis-Defect von ca. $4 \mathrm{~cm}$ Durchmesser. An der Aussenseite des Unterschenkels oberhalb des Malleolus ext. ist die Hant anästhetisch. Sonst Sensibilität überall erhalten. Die Bewegungen, die Patient im Kniegelenk auszuführen vermag, haben an Ausgiebigkeit stetig zugenommen. Patient tann das Bein mit einiger Mühe bis zu einem Winkel von ca. $120^{\circ}$ flectiren. Bewegungen im Fussgelenk gleichfalls activ möglich. Patient kann mit einem Stock obne Beschwerden gehen. Allgemeinbefinden normal. Patient wird $z a$ weiterer privater Behandlung nach seinem Wohnort (ausserhalb Rigas) entlassen. -

Die Geschwulst, welche sich nur in einzelnen Stücken entferneu liess, zeigt makroskopisch den typischen Bau eines Cavernoms: die bekannte schwammartige, grobhöckerige Form. Auf dem Durchschnitt sieht man zahlreiche Lumina, die strotzend mit Blut gefüllt sind. Während einzelne durch ihre melarschichtige Wandung und kreisrunde Form sich als Blut- 
gefässe documentiren, zeigen die mèisten weit grössere Ausdehnung, unregelmässige Gestalt und dünne Wandung, sind also Cavernen im üblichen Sinne. All diese Lumina sind durch Züge von Fett, Bindegewebe oder Muskelgewebe untereinander verbunden.

Mikroskopisch bestehen die Geschwulsttheile, je nachdem, ob sie aus dem Unterhautzellgewebe oder Muskelgewebe stammen, aus einem in welligen Schichten binziehenden derben Bindegewebe mit eingesprengten Zügen von Fettgewebe, oder aus Muskelgewebe in Zustande der Atrophie: die Fasern sind geschrumpft, an manchen Stellen geschlängelt, ohne sichtbare Kerne, durch eingelagertes Fett auseinander gedrängt. In dieses Gewebe eingelagert finden sich nun überaus zahlreiche Blutmengen, von wenigen aneinander gelagerten Blutkörperchen bis zu grossen, compacten Haufen. Und zwar lässt sich in der Art, wie sie sich in die Gewebe eingelagert haben, deutlich ein zwiefacher Modus erkennen:

Einmal finden sich die Blut-Ansammlungen innerhalb von Höhlungen, welche von einer Wand ausgekleidet sind, also in Cavernen. Die Wand dieser Cavernen besteht in einer Lage von Zellen mit dicken, ovoiden, an anderen Stellen platt spindelförmigen Kernen. Die Cavernen sind theils einkammerig, theils von bindegewebigen Septen in verschiedener Dicke durchzogen, wodurch ein Fächerung und Eintheilung in viele kleine Hohlräume geschieht. An anderen Stellen ragen bindegewebige Riffe und Zacken in das Lumen hinein, als Ausdruck ehemaliger, nunmehr geschwundener Septen. Dass diese Cavernen als runde Hohlräume schon längere Zeit bestehen, dafür spricht der Umstand, dass das der Wand angrenzende Bindegewebe eine concentrische Anordnung erlangt hat.

Die zweite Form, wie sich die Blutmengen dem Auge darstellen, ist eine von der eben beschriebenen durchaus verschiedene:

Man sieht nehmlich kleinere und grössere Mengen obne jede Wandung frei im Gewebe liegen, und zwar als compacte Hämatome zwischen den auseinander gewichenen Gewebsschichten, also in Gewebsspalten, oder analog einer kleinzelligen Infiltration, diffus zwlsehen den Zellen. -

Ich habe das Cavernom in dieser soeben von mir geschilderten Art nirgends beschrieben gefunden. Von Virchow an, der die Darstellung des Angioma cavernosum in klinischer und anatomischer Hinsicht am erschöpfendsten gegeben, wird von allen Autoren das Hauptgewicht auf die Cavernen-Bildung gelegt und ausschliesslich von Cavernen gesprochen, als von Hohlräumen, welche eine Wand besitzen in Form eines Plattenepithels. Virchow giebt sogar an, dass dieses Plattenepithel nur in Fällen fauliger Zersetzung nicht in situ ist" ${ }^{17}$ ).

1) Virchow, Die krankhaften Geschwülste. 
Volkmann ${ }^{1}$ ) spricht, bei der Beschreibung eines aus mehreren Cysten bestehenden Cavernoms, von der inneren Auskleidung dieser Cysten als einer Schicht platter, spindelförmiger bis polygonaler Zellen mit Kernen, die sich ganz wie GefässEndothelien verhielten.

In einem kürzlich von $B e h r^{2}$ ) veröffentlichten Fall von ausserordentlich grossem Cavernom der Lumbalgegend, beschreibt dieser die Hohlräume als mit einem glatten Epithel ausgekleidet.

Weniger bestimmt äussert sich $L \ddot{\text { ücke }}{ }^{8}$ ) in seiner Darstellung des cavernösen Angioms von den Cavernen, die gewöhnlich eine Epithel-Auskleidung haben sollen, ähnlich dem Gefäss-Epithel.

Nur einmal, in einem von Hildebrand $t^{4}$ ) veröffentlichten Falle, habe ich die Bemerkung gefunden, dass eine Auskleidung der Hohlräume mit Epithel nicht mit Sicherheit nachgewiesen werden konnte. Doch führt auch Hildebrandt diesen Umstand nur an, ohne daraus weitere Schlüsse zu ziehen.

Was nun meinen Fall anbelangt, so möchte ich mich nieht damit begnügen, die Häufigkeit, mit der das Blut in wandungslosen Gewebsspalten erscheint, einfach anzuführen, sondern möchte mich der Ansicht zuneigen, dass gerade diesem Umstande eine grosse Bedeutung zukommt, und zwar insofern, als das Stadium, in welchem bei dem Cavernom das Blut in wandungslosen Räumen fliesst, ein Glied in der Entwickelungsgeschichte dieser Geschwulst darstellt, und die Verwerthung dieser Erscheinung möglicher Weise eine durchaus einfache und befriedigende Lösung der Frage nach dem Wesen der Geschwulst zu ergeben vermag.

Bevor ich mich über meinen Gedanken näher ausspreshe, möchte ich, der Uebersicht halber, die bis jetzt existirenden Ansichten über die Entstehung des cavernösen Angioms anführen:

Virchow nimmt bei der Entstehung der Cavernome zwei Stadien an, eines der Gefässbildung und eines der Erweiterung

1) Langenbeck, Archiv 15.

2) Dissertation, Kiel 1900.

3) Pitha und Billrotb, Handb. d. Chir.

4) D. Zeitschr. f. Chir. 30. 
der Gefässe und Umbildung zu Hohlräumen, und stellt als Ursache der Vergrösserung eine fortschreitende Reizung dar, welche im Umfang der Geschwulst auftritt und das Nachbargewebe zur Granulation veranlasst. Dieses granulirende Gewebe (Matrix) erzeugt neue Gefässe, in welche das Blut von den alten her eindringt. Dadurch werden die neuen Gefässe mehr und mehr ausgedehnt und zu Schlängelung und Windung mit seitlicher Ausdehnung veranlasst. Inzwischen verschwindet das $Z$ wischengewebe durch Atrophie und wird usurirt. Eine Bildung von Capillargefässen findet dabei nur in geringem Maasse statt. Die neuen Gefässe zeigen schon früh mehrfach geschichtete Wandungen, so dass sie, je nach den Umständen, bald mehr arteriell, bald mehr venös erscheinen. Die Ausdehnung der Gefässe ist nicht einfache Dilatation, dann müsste Verdünnung der Wand vorhanden sein. Man findet auch Gefässräume mit grossem Lumen und dicker Wand. Es ist also eine Dilatation mit Hypertrophie, (Ektasie mit Hyperplasie). Diese die Ektasie begleitende Hyperplasie, welche in progressiver Vermehrung und Ausbildung von Wandel-Ementen besteht, ist ein durchaus activer Vorgang.

Volkmann hält die in dem von ihm beschriebenen Falle vorhandene Auskleidung der Cysten mit Endothel für einen Beweis ihrer Herkunft von Gefässen. Er führt an, dass die Umwandlung von cavernösen Bluträumen zu Cysten schon mehrfach beobachtet sei, ebenso wie er die Entstehung von Cysten direct aus Venen für erwiesen hält.

Rindfleisch ist der Ansicht, dass ein Cavernom sich aus jedem mit Blutgefässen versehenen Gewebe entwickeln könne, und nennt die Entstehung cavernöser Geschwülste "Cavernöse Metamorphose", die zu Stande kommen soll durch fibroide Degeneration des capillären Abschnittes der Blutbahn. Nach ihm ist der Vorgang eine Ausdehnung der Gefässe durch Schrumpfung des Nachbargewebes.

Nach der Ansicht dieser Autoren sind also die Cavernen ehemalige, nunmehr bloss umgewandelte Gefässe, und circulirt das Blut, in welchem Entwickelungs-Stadium sich auch das Cavernom befinden möge, ausschliesslich innerhalb vorgebildeter, mit Wandung versehener Räume.

Erst bei Lücke findet sich eine andere Auschaung. Er 
wirft die Frage auf, ob nicht etwa das Blut sich von den vorhandenen Gefässen aus in den Geweben neue Bahnen bildet, die vielleicht in den Lymphräumen oder anastomosirenden Zellen vorgezeichnet sind, beziehungsweise ob nicht ein Cavernom dadurch entstehen kann, dass das Blut aus den Gefässen heraus in das Nachbargewebe eindringt, dessen Schichten auseinander drängend.

Diese von Lücke aufgeworfene Frage möchte ich nun auf Grund meines Befundes mit der Behauptung vervollständigen: dass ein Cavernom dadurch entsteht, dass primär, nach Ruptur von Gefässen, es zu freien Blutergüssen in das Gewebe kommt, und dass secundär von diesem Gewebe, als Reaction auf den Reiz, die dem Hämatom zunächst liegende Bindegewebs-Zellschicht sich in die vielfach besprochene Endothelwand verwandelt, mit dieser den Bluterguss von allen Seiten umgiebt und so eine Caverne bildet. -

Den Befund meiner Präparate, der mich zu dieser Behauptung veranlasste, habe ich in den beigefügten Abbildungen wiedergegeben. Nach denselben lassen sich drei aufeinander folgende Stadien unterscheiden, in denen das Blut sich dem Auge darbietet:

1. Das Blut befindet sich noch in geschlossenen Gefässbahnen (Taf. VII Fig. 1).

2. Das Blut hat die Gefässbahn verlassen; es liegt frei als zusammenbängendes Extravasat (Hämatom) im auseinander gedrängten Gewebe (Taf. VII Fig. 2, 3, 4), oder die einzelnen Blutkörperchen liegen wach Art der kleinzelligen Infiltration diffus zwischen den Zellen (Taf. VII Fig. 5).

3. Die Blutmassen liegen in mit deutlichen Wandungen versehenen Cavernen (Taf. VII Fig. 4, 6).

Meine Behauptung möchte ich nunmehr mit folgenden Argumenten stützen:

Dass das Blut eines Cavernoms aus dem Gefässsystem stammt, bedarf keiner weiteren Erörterung. Dass es ein Stadium giebt, in welchem das Blut, nachdem es das Gefäss verlassen, frei und wandungslos im Gewebe liegt, geht aus meinen Abbildungen 2, 3,5 hervor. Gegen die Möglichkeit, dass die Endothelschicht 
übersehen worden ist, spricht der Umstand, dass die EndothelAuskleidung in Fig. 4 und 6 bei der gleich starken Vergrösserung absolut deutlich ist, und dass in Fig. 4 beide Stadien nebeneinander sichtbar sind, rechts der keilförmig auseinander gedrängte Bindegewebsraum, links eine ovoide Caverne mit Endothel und deutlichen Kernen. In Fig. 5 hat das Blut sich noch keinen Hohlraum geschaffen, sondern diffus das Gewebe durchsetzt.

In Fig. 2 und 3 hat sich das Blut zwischen Bindegewebsschicht und Fettschicht, beziehungsweise zwischen die einzelnen Fettzellen gedrängt; wäre hier eine Endothelschicht vorbanden, so miisste sie mit derselben Deutlichkeit sichtbar sein, mit der sich die übrigen Zellgrenzen differenziren lassen.

Dass man in all diesen Fällen von Cavernen nicht sprechen kann, sondern dass es sich um Spalten handelt, welche durch Auseinanderweichen der Gewebsschichten entstanden sind, dafür spricht ferner der Umstand, dass die Bindegewebsfasern unbehindert um das eingedrungene Blutquantum in der alten Richtung weiterziehen (Taf. VII Fig. 2, 4), ja stellenweise den Eindruck machen, als ob sie in ihrer Continuität zerrissen sind (Taf. VII Fig. 1), während im fertig ausgebildeten Cavernen-Stadium in Folge längeren Bestehens der Blutmenge an dieser Stelle das umgebende Gewebe Zeit gefunden hat, in seiner Structur sich der Form des Hämatoms anzupassen, und daher überall eine mehr oder weniger deutliche concentrische Schichtung zeigt.

Allgemein wird angegeben, dass Cavernome angeboren kaum je vorkämen, sondern dass ihnen ein teleangiektatisches Stadium vorausgehe. Ich glaube auch, dass sich nichts dagegen einwenden lässt, die Teleangiektasie als Vorstufe des Cavernoms zu betrachten, ebenso wie sich kaum bestreiten lassen wird, dass aus einem Cavernom durch zunehmende Ausdehnung und Schwund der Septa eine Blutcyste entstehen kann, nur scheint mir unwahrscheinlich, dass die Hohlräume des Cavernoms nur erweiterte, im übrigen aber in ihrer Continuität erhaltene Blutgefässe darstellen sollen. Es ist viel complicirter, sich vorzustellen, dass das Endothel der Cavernen, als alleiniges Ueberbleibsel einer ehemaligen Gefässwand, von dieser herstammt, als den viel einfacheren Vorgang anzunehmen, dass die innerste Schicht des 
Bindegewebsspaltes, in welchen das Blut gedrungen ist, sich zu einer Endothel-Lage umgewandelt hat; denn die Endothelzellen sind umgewandelte Bindegewebszellen.

Das Blut spielt, wenn es aus den Gefässen in das Gewebe gedrungen ist, die Rolle eines heterologen Elementes, eines Fremdkörpers; und allen Geweben gemeinsam ist das Bestreben, gegen die directe Berührung oder ein weiteres Vordringen des Fremdkörpers Maassregeln zu ergreifen durch Errichtung einer Schutzwand. Ich glaube daher, dass der Endothelwand um die einzelnen Hämatome dieselbe Rolle zukommt, wie allen $\mathrm{Ab}$ kapselungs-Processen um längere Zeit danernde, entzündliche Heerde, oder um wirkliche, nicht resorbirbare Fremdkörper. Ich glaube ferner, dass die Endothelkapseln der Cavernen im Kleinen nichts Anderes darstellen, als die vielfach beschriebene, derbe Bindegewebs-Kapsel, welche die gesammte cavernöse Geschwulst gegen ihre Umgebung abgrenzt und einem weiteren Vordringen derselben ein Ziel setzen soll.

Nach diesen Ausführungen, und wenn man sich die beschriebenen Umstände zu einer Kette aneinander reiht, fehlt aber auch zum Verständniss der Entstehungsursache des Angioma cavernosum nur Eins, nehmlich die Beantwortung der Frage: aus welchem Grunde kommt es zu den spontanen Blut-Austritten in das Gewebe, und warum persistiren diese Hämatome im Gewebe, ist doch normaler $W$ eise das Geschick eines ins Gewebe gelangten Blutergusses das, in kürzerer Zeit durch Resorption wieder zu verschwinden, indem seine Bestandtheile dem Kreislauf wieder zugeführt oder vom umgebenden Gewebe aus substituirt werden.

Zur Beantwortung dieser Frage lässt sich aber unschwer die Annahme einer angeborenen Entwickelungsstörung eines bestimmten Gewebsbezirks, eine Hypoplasie der Gewebselemente, heranziehen. Ebenso, wie es ein Zurückbleiben im Grössenwachsthum ganzer Organe giebt, so mag es ein Zurückbleiben in Bezug auf die übrigen Lebensäusserungen von Zellgruppen geben, und dadurch eine mangelhafte Elasticität, eine leichte Zerreisslichkeit, eine geringere Neigung zur Restitutio ad integrum und schliesslich eine geringe Resorptions-Fähigkeit bedingt sein.

Welcher Art die Schädigungen sind, welche die Zellen im intrauterinen Leben treffen, wird sich nie sicher constatiren 
lassen. Anzunehmen ist, dass diejenigen Zellgruppen, wélche am längsten in der Entwickelung verharren, am ehesten die Zeichen der Hypoplasie tragen werden, weil sie sich am längsten in einem wenig widerstandsfähigen Stadium befinden. Als Beleg dieser Annahme könnte man vielleicht die von Virchow so benannten fissuralen Angiome anführen, die an Kopf und Hals, an solchen Stellen auftreten, wo es zu einem Schluss der embryonalen Kiemenspalten gekommen ist - weniger wegen des Gefässreichthums dieser Bezirke, als vielleicht gerade wegen einer ungenügenden Entwickelung der hier befindlichen Zellgruppen. Die ungenügende Entwickelung mag ihren Grund darin haben, dass die Kiemenspalten diejenigen Theile sind, in welchen der Entstehungs-Process mit am längsten dauert und am spätesten beendet ist.

Wie ich oben angeführt habe, wird ziemlich allgemein angenommen, dass das Cavernom sich aus einer einfachen Teleaniektasie entwickelt. Ebenso ist mehrfach der Nachweis geliefert von dem Uebergang des Cavernoms zur Blutcyste. Stimmt man mit diesen Anschauungen überein, so ergiebt sich eine ziemlich einfache Lösung der ganzen Angiomfrage, indem die einzelnen, bisher getrennten Formen nicht etwas Besonderes darstellen, sondern nur verschiedene Stadien eines und desselben pathologischen Vorganges sind. Derselbe würde, wie folgt, vor sich gehen:

I. Der normale Blutdruck findet in Folge mangelhafter Elasticität der Gefässwände und des Gewebes ungenügenden Gegendruck, weitet die Capillaren und kleinsten Venen aus Teleangiektasie.

II. Durch Einwirkung eines Trauma oder durch fortgesetzte mechanische Irritation kommt es zur Berstung der stark gedehnten Gefässwände und zu Blut-Austritten ins Gewebe. Es tritt als neues Moment hinzu die Reaction des Gewebes, die Wandbildung - Cavernom.

III. Der Blutdruck erweist sich als stärker, als die Reactionskraft des Gewebes. Durch fortgesetzten Zufluss neuer Blutmengen unterliegen die Interstitien starkem Druck, sie werden zu dünnen Septen und schwinden schliesslich ganz. Der Endeffect ist die Blutcyste. 
Die Heilungen, die man in jedem dieser drei Stadien des Angioms beobachtet hat, lassen sich bei der einfachen Teleangiektasie wohl durch nachträgliche Stärkung der Widerstandskraft der Gewebe erklären. Hier ist eine Restitutio ad integrum möglich. Im Cavernom- und Cysten-Stadium können Abkapselung und der bekannte Vorgang der Thromben-Bildung mit Organisation den Process zum Stillstand bringen, doch sind hier die Bedingungen weit schlechtere, da der Bluterguss ausserhalb des Gefässes noch weniger Widerstände vorfindet, die seiner Ausbreitung Einhalt thun könnten, als in dem intacten Gefässrohr. -

Zum Schluss möchte ich noch eine Veröffentlichung $\mathrm{Neu}$ mann's aus dem Jahre 1861 erwähnen'), die mir erst nach Beendigung dieser Arbeit zugänglich wurde, und die mir insofern von höchstem Interesse ist, als $\mathrm{N}$ e umann bei der Untersuchung eines aus cavernösem Gewebe bestehenden Nasenrachen-Polypen demselben Befund begeguete, wie ich in meinem Falle. Er sah in den Interstitien, zwischen den Gefässdurchschnitten, sowie an den Uebergangsstellen zwischen dem cavernösen und dem einfach fibrösen Gewebe ein mit Blutkörperchen erfülltes Netzwerk, und fand das Blut in Canälen, „die, einer eigentlichen Wand entbehrend, unmittelbar von der Intercellularsubstanz begrenzt wurden".

Anch Neumann leitet die Anfüllung der Zellen mit Blut indirect von einem Extravasat oder direct aus der offenen Communication der Gefässlumina mit den Zellen her, spricht sich jedoch über die Beziehung dieses Vorgangs zur CavernomBildung nicht weiter aus. -

Meinem Chof, Herrn Dr. Paul Klemm, danke ich für die freundliche Ueberlassung des Materiales, sowie für die Anregung zu obiger Arbeit.

1) Dieses Archiv Bd. 21. 